$\mathrm{N}$ must be found iteratively. A computer program was written to find $\mathrm{N}$ for a range of values of the various factors, and the results are presented in a table.

The use of the table can be illustrated as follows : There breeds are to be evaluated, chiefly for growth rate, which has a heritability of .3 and a phenotypic coefficient of variation of a bout 10 per cent. If the required repeatability of the progeny test is .8 , then 22 progeny per bull are needed. To have a probability of .8 of detecting a difference of 2 per cent of the mean at the .05 level will require 11 bulls per breed, or an experiment of $3 \times 11 \times 22=726$ cattle in total. With a relaxed significance level of $.10,7$ bulls per breed will suffise, requiring an experiment with $3 \times 7 \times 22=462$ cattle.

\title{
DIE WIDERSTANDSF ÄHIGKEIT DER RINDERKLAUEN, EIN ÖKONOMISCHER FAKTOR DER INDUSTRIELLEN MILCH- UND FLEISCHPRODUKTION
}

H. O. GRAVERT und S. CAMARA. - Bundesanstalt für Milchforschung, Kiel, BRI).

Von 605 geschlachteten Rindern wurde an je 8 Klauen der Abrieb auf einer Betonfläche bestimmt. Die Heritabilität des Abriebs betrug nur $h^{2}=0,20$, berechnet aus 30 Halbgeschwistergruppen mit 281 Jungbullen, die unter gleichen Umweltbedingungen standen. Der Abrieb war auf nassem Beton um $83 \%$ grösser als auf trockenem Beton. Der Zementanteil im Beton hatte keinen Einfluss, die Korngrösse der Beimengungen nur einer geringen. Dagegen wirkte sich die Struktur der Beimengungen wesentlich aus, $z$. B. war der Abrieb auf Tuffstein etwa doppelt so gross wie auf Quarzsand. Eine Behandlung der Klauen mit Formalin oder Branntkalk reduzierte den Abrieb um 6 bzw. $24 \%$. - Die experimentellen Ergebnisse konnten in Versuchen mit lehenden Kühen in der Tendenz bestätigt werden. 